${ }^{1}$ Departamento de Nefrología. Escuela de Medicina. Facultad de Medicina. Pontificia Universidad Católica de Chile. Santiago, Chile.

2Programa de Farmacología y Toxicología. Escuela de Medicina. Facultad de Medicina. Pontificia Universidad Católica de Chile. Santiago, Chile.

${ }^{3}$ Departamento de Medicina Intensiva. Escuela de Medicina. Facultad de Medicina. Pontificia Universidad Católica de Chile. Santiago, Chile.

4Departamento de Anatomía Patológica. Escuela de Medicina. Facultad de Medicina. Pontificia Universidad Católica de Chile. Santiago, Chile.

ástudiante Escuela de Medicina. Facultad de Medicina. Pontificia Universidad Católica de Chile.

Trabajo no recibió financiamiento. Los autores declaran no tener conflictos de interés.

Recibido el 15 de septiembre de 2020, aceptado el 22 de junio de 2021.

Correspondencia a:

Dr. Eduardo Ávila

Departamento de Nefrología. Escuela de Medicina. Facultad de Medicina. Pontificia Universidad Católica de Chile. Santiago, Chile. eravila@uc.cl

\section{Nefropatía aguda por fosfatos secundaria al uso de laxantes con fosfato de sodio: caso clínico-patológico}

\author{
EDUARDO ÁVILA ${ }^{1}$, DALAY PURTO ${ }^{a}$, SOFÍA KUTSCHER ${ }^{a}$, \\ CRISTINA CORNEJOa, NICOLÁS SEVERINO ${ }^{2,3}$, \\ GONZALO P. MÉNDEZ ${ }^{4}$, RODRIGO TAGLE ${ }^{1}$
}

\section{Acute phosphate nephropathy secondary to the use of oral sodium phosphate laxatives. Report of one case}

Acute phosphate nephropathy (APN) is an acute renal failure secondary to the use of oral sodium phosphate (OSP) laxatives, with a high risk of progression to chronicity. We report a 60-year-old woman with mixed connective tissue disease whose serum creatinine increased up to $2.0 \mathrm{mg} / \mathrm{dL}$ in her regular control tests, without an evident causative factor. Kidney biopsy showed numerous intratubular calcium phosphate deposits, consistent with APN. She had a history of OSP laxative intake, and a sodium phosphate enema was used before a colonoscopy performed six months earlier. The temporal association between the use of OSP laxatives and acute kidney injury, should lead to the suspicion of APN. The urine sediment is generally normal or with mild to moderate proteinuria. The diagnosis is confirmed with a kidney biopsy. Until now, there is no specific treatment for $A P N$, thus prevention is essential. In high-risk patients for developing APN, the administration of these laxatives should be avoided.

(Rev Med Chile 2021; 149: 934-938)

Key words: Acute Kidney Injury; Laxatives.
E 1 año 2014 la Food and Drug Administration publicó una alerta de falla renal, arritmias y muerte asociada al uso de laxantes de fosfato de sodio oral (FSO), recomendando precaución en su uso ${ }^{1}$.

La nefropatía aguda por fosfatos (NAP) es un tipo de falla renal aguda (IRA) secundaria al uso de laxantes de FSO, generalmente en contexto de preparación para colonoscopías o resolución de fecalomas. El daño renal inducido por FSO puede producir una enfermedad aguda con posible evolución hacia la enfermedad renal crónica (ERC) ${ }^{2}$.

El propósito de este trabajo consiste en alertar sobre el uso de FSO en pacientes con factores de riesgo a través de la presentación de un caso clínico-patológico.

\section{Caso clínico}

Mujer de 60 años con enfermedad mixta del tejido conectivo, hipertensión arterial (HTA) bien controlada. En tratamiento con micofenolato, hidroxicloroquina y valsartán. En exámenes de control se pesquisa creatinina plasmática hasta $2,0 \mathrm{mg} /$ $\mathrm{dL}$ (basal $0,7 \mathrm{mg} / \mathrm{dL}$ ), sin un factor evidente.

En laboratorio destaca examen de orina sin proteinuria, leucocituria, eosinofiluria ni hema- 
turia significativas, con presencia de cristales de ácido úrico abundantes. Electrolitos plasmáticos, calcemia y fosfatemia normales. Hematocrito $27 \%$, sin eosinofilia y VHS $25 \mathrm{~mm} / \mathrm{h}$. Electroforesis de proteína en sangre y orina sin peak monoclonal. Serología inmunológica, complementos C3 y C4 normales, anticuerpos: ANA 1/320 patrón centrómero, Anti RNP 133,4 UI, Anti DNA 35,4 UI (valor normal <30), Anti Sm, Anti MPO, Anti PR3, anticardiolipinas, anti B2-Glicoproteína I y anticoagulante lúpico negativos.

Ecografía doppler renal sin estenosis de arteria renal, riñones de tamaño normal con aumento de ecogenicidad y leve disminución del espesor cortical. Tomografía computada renal sin contraste, no mostró litiasis ni calcificaciones.

Dado la falla renal reciente sin causa aparente ni laboratorio especifico se realiza biopsia renal que mostró en la microscopía óptica, numerosos depósitos de fosfato cálcico en segmentos tubulares distales, con signos de daño secundario (Figura 1). Dicha muestra contuvo 15 glomérulos, ninguno obsoleto y todos de morfología conservada. La atrofia tubular y fibrosis fue de $20 \%$. Los exámenes
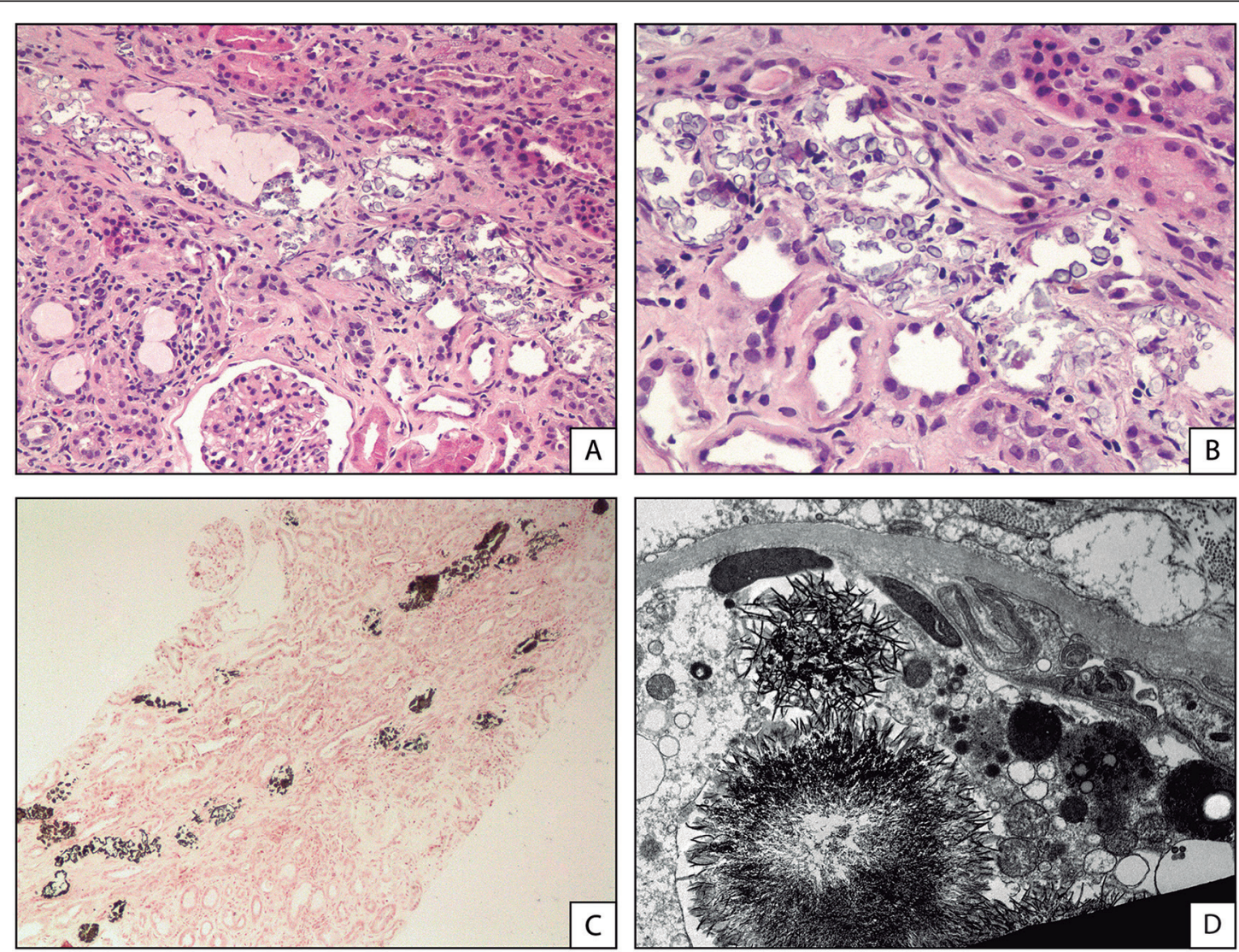

Figura 1. A, B, C: Microscopía óptica. A. Zona de corteza renal con signos de daño tubular agudo en segmentos rectos distales y colectores, con túbulos distendidos irregularmente que contienen numeroso material basófilo, con signos de ectasia de proteína Tamm Horsfall. Existen signos de atrofia por túbulos adyacentes disminuidos de diámetro, fibrosis intersticial adyacente e inflamación crónica (Tinción de hematoxilina y eosina; 200x). B. Segmentos rectos distales que contienen numerosas concreciones cálcicas basófilas redondeadas, e agregados intracitoplasmáticos e intraluminales, con descamación epitelial secundaria (Tinción de hematoxilina y eosina; 400x). C. Área de corteza que muestra depósito tubular masivo de calcificaciones con tinción negruzca intensa, compatibles con fosfatos de calcio (Tinción de von Kossa; 100x). D. Microscopía electrónica. Sección transversal de túbulo distal con daño agudo que incluye descamación epitelial y presencia de lisosomas prominentes. Se reconocen dos calcificaciones constituidas por agregados masivos de espículas electron-densas dispuestas radialmente, generando capas de distinta densidad en relación a un eje central (Tinción acetato de uranilo-citrato de plomo; 8.200x) 
de inmunofluorescencia y microscopía electrónica mostraron además, de manera concurrente, depósitos mesangiales mínimos de IgA. Los hallazgos fueron compatibles con una NAP con signos de cronicidad túbulo intersticial leve.

Posteriormente se rescata antecedente de uso de 2 frascos de laxantes de FSO y 1 fleet enema de fosfato de sodio (FS) previo a colonoscopía 6 meses antes.

Se obtuvo consentimiento informado de la paciente para la publicación.

\section{Discusión}

El FSO es un laxante salino que requiere poco volumen para conseguir una limpieza del colon, siendo igual de efectivo y mejor tolerado que la solución de polietilenglicol (PEG). Su efecto radica en ser una sustancia osmótica hiperosmolar ${ }^{3,4}$.

El caso descrito representa un ejemplo típico de nefropatía por FSO; un paciente con falla renal sin otro agente etiológico evidente, con hallazgos específicos en la biopsia y la posterior asociación con la administración de laxante de FSO.

La clínica de la enfermedad describe dos formas de presentación, una aguda reversible y otra de inicio tardío generalmente irreversible. La forma aguda se produce a las pocas horas de la administración de FSO y ocurre en el contexto de hiperfosfatemia aguda, que puede asociarse a hipocalcemia. El compromiso renal agudo suele pasar desapercibido a menos que se presenten signos de hipocalcemia como arritmias o parestesias, y por lo general no se obtiene una biopsia renal.

En cambio, la NAP de inicio tardío, como en este caso, generalmente ocurre en sujetos asintomáticos días a meses posterior a la administración de FSO, por lo que las concentraciones de fósforo y calcio son ya normales cuando se descubre la falla renal.

En un estudio de la Universidad de Columbia ${ }^{5}$ se evaluaron 21 pacientes con NAP identificados por biopsia renal. El $60 \%$ presentó sedimento de orina anodino, $23 \%$ leucocituria y $14 \%$ hematuria. La excreción de proteínas posterior a las $24 \mathrm{~h}$ de administración fue menor a $600 \mathrm{mg}$ /día en 13/14 participantes, siendo infrecuente proteinuria mayor a $1 \mathrm{~g}$. La mayoría de estos pacientes recibió una dosis adecuada de FSO y no presentaban contraindicaciones para su uso. En el mismo estudio, se describe en promedio una creatinina de $3,9 \mathrm{mg} /$ $\mathrm{dL}$ al mes de seguimiento. A los 16,7 meses el 19\% presentó ERC terminal, el resto de los pacientes tuvieron en promedio una creatinina de $2,4 \mathrm{mg} /$ dL y ninguno volvió a su función renal basal, concordante con otros reportes donde la función renal parece no recuperarse $e^{6-8}$.

En la década de 1990 se comenzó a usar FSO como laxante para lograr colonoscopías óptimas. Desde entonces han sucedido varios reportes de IRA en relación a su uso ${ }^{1}$. La ingesta de fósforo en la dieta es aproximadamente $1 \mathrm{~g}$ al día, no obstante, algunos laxantes de FSO comercializados en Chile tanto en formulación oral o enema, contienen hasta $13 \mathrm{~g}^{9}$ (Tabla 1 ).

No existen datos de incidencia de la NAP. Algunos estudios han estimado la reducción de la VFG a los 6 a 12 meses posterior al uso de $\mathrm{FSO}^{10-12}$. Uno de éstos, con 9799 participantes, mostró que la IRA (definida por elevación de creatinina $>50 \%$ ) posterior a una colonoscopía se presentó en el $1,2 \%{ }^{11}$. Otro estudio retrospectivo de 2237 pacientes que se realizaron colonoscopía ambulatoria, presentó 6,3\% de IRA a los 6 meses $^{13}$.

Dentro de la teoría que explica esta patología, se describe la ocurrencia de un aumento transitorio de la fosfatemia; así, el 28\% de los pacientes presentaron niveles de fosfato $>8 \mathrm{mg} / \mathrm{dL}$ después de la administración de $\mathrm{FSO}^{14}$. Lo anterior refleja un alto nivel de absorción intestinal de fosfato en corto tiempo debido a una lenta regulación de los transportadores intestinales de fosfato en comparación con los renales ${ }^{15,16}$.

Histopatológicamente, los hallazgos presentados concuerdan con las biopsias renales de NAP, que muestran depósito generalizado de fosfato de calcio en la luz tubular, daño obstructivo, lesión epitelial directa e inflamación, y depósito en intersticio peritubular ${ }^{17}$. El daño directo ocurre en todos los segmentos del nefrón, sin embargo, el depósito ocurre característicamente en los segmentos distales, favorecido por la menor reabsorción proximal de fósforo debido a la hiperfosfatemia existente. Los depósitos de fosfato de calcio se logran distinguir por la tinción de von Kossa, que destaca los fosfatos.

Una biopsia temprana a la administración de FSO muestra cambios degenerativos tubulares similares a necrosis tubular aguda. En cambio, una biopsia más tardía puede mostrar cambios crónicos, con atrofia tubular, fibrosis intersticial, 
Tabla 1. Presentaciones comerciales de compuestos que incluyen fósforo

\begin{tabular}{|c|c|c|c|c|c|c|c|c|}
\hline \multirow{2}{*}{$\begin{array}{l}\text { Presentación } \\
\text { comercial }\end{array}$} & \multicolumn{7}{|c|}{ Compuesto (g) } & \multirow{2}{*}{$\begin{array}{l}\text { Fósforo } \\
\text { elementa } \\
\text { (g) }\end{array}$} \\
\hline & $\mathrm{NaH}_{2} \mathrm{PO}_{4}$ & $\begin{array}{l}\mathrm{NaH}_{2} \mathrm{PO}_{4} \\
\quad \mathrm{H}_{2} \mathrm{O}\end{array}$ & $\begin{array}{l}\mathrm{NaH}_{2} \mathrm{PO}_{4} \\
{ }^{2} 2 \mathrm{H}_{2} \mathrm{O}\end{array}$ & $\mathrm{Na}_{2} \mathrm{HPO}_{4}$ & $\begin{array}{l}\mathrm{Na}_{2} \mathrm{HPO}_{4} \\
* 7 \mathrm{H}_{2} \mathrm{O}\end{array}$ & $\begin{array}{c}\mathrm{Na}_{2} \mathrm{HPO}_{4} \\
* 12 \mathrm{H}_{2} \mathrm{O}\end{array}$ & $\mathrm{H}_{3} \mathrm{PO}_{4}$ & \\
\hline Edoxil enema adulto & & 10,00 & & & & 8,00 & & 2,94 \\
\hline $\begin{array}{l}\text { Fosfato de sodio enema } \\
\text { adulto, fleet }\end{array}$ & & & & 10,01 & & & 6,58 & 4,26 \\
\hline $\begin{array}{l}\text { Forflow solución para } \\
\text { enema }\end{array}$ & & 16,00 & & & 6,00 & & & 4,28 \\
\hline Casen enema adultos & & & 18,10 & & & 8,00 & & 4,29 \\
\hline Hofsamed enema & & & 18,20 & & 5,93 & & & 4,30 \\
\hline $\begin{array}{l}\text { Bloner solución para } \\
\text { enema }\end{array}$ & & 3,18 & & 16,00 & & & & 4,31 \\
\hline Phosfoenema solución & 16,00 & & & 6,00 & & & & 5,44 \\
\hline $\begin{array}{l}\text { Fosfosoda solución } \\
\text { oral }\end{array}$ & & & 24,40 & & & 10,80 & & 5,78 \\
\hline Fleet enema & 19,00 & & & 7,00 & & & & 6,43 \\
\hline Fosfo-dom solución oral & & 48,00 & & & 18,00 & & & 12,85 \\
\hline Gadolax solución oral & & 48,00 & & & 18,00 & & & 12,85 \\
\hline
\end{tabular}

$\mathrm{H}_{3} \mathrm{PO}_{4}$ : ácido fosfórico; $\mathrm{NaH}_{2} \mathrm{PO}_{4}$ : fosfato monosódico; $\mathrm{Na}_{2} \mathrm{HPO}_{4}$ : fosfato disódico; $\mathrm{KH}_{2} \mathrm{PO}_{4}$ : fosfato monopotásico; $\mathrm{K}_{2} \mathrm{HPO}_{4}$ : fosfato dipotásico.

inflamación e infiltrado linfocitario y de neutrófilos, similar al caso presentado (Figura 1).

En la serie de la Universidad de Columbia, se lograron identificar ciertos factores de riesgo para el desarrollo de NAP ${ }^{5}$. Éstos fueron: dosis y frecuencia de FSO, ERC, hipovolemia, fármacos que bloquean el sistema renina-angiotensina (SRA), edad avanzada y género femenino. En otros estudios se identificaron adicionalmente: HTA, diabetes mellitus, uso de diuréticos, antiinflamatorios no esteroidales y litio ${ }^{2,5}$.

El $67 \%$ de los que presentaron NAP utilizaban fármacos que bloquean el SRA ${ }^{10,13}$. La edad avanzada se asoció sin lograr precisar una edad determinada. Así mismo, el género femenino se relaciona probablemente por su menor peso y menor VFG respecto a los hombres ${ }^{5,13}$. En otro estudio se detectó como factor de riesgo la alteración del tránsito intestinal ${ }^{16}$.

La hipovolemia genera mayor absorción proximal de sodio, determinando en túbulo distal aumento de la concentración de fosfato y aumento de la adherencia de cristales en epitelio.

La dosificación de fosfato es directamente proporcional al daño; así, la mayor concentración o frecuencia de administración de FSO se asocia a mayor grado de lesión ${ }^{5}$. Este caso recibió una dosis total de fósforo de 17,9 g.

La forma de administración también ha sido estudiada. La mayoría de los estudios han asociado la NAP al uso de estos laxantes vía oral. Sin embargo, algunos estudios han descrito casos de NAP por el uso de enemas de FS con dosis desde $250 \mathrm{ml}$, con mayor riesgo en adultos mayores ${ }^{18}$. En otro estudio retrospectivo realizado en 70.499 participantes que se realizaron colonoscopía, el uso de enema de FS se asoció a un mayor riesgo de IRA dentro de los 15 meses posteriores a su uso ${ }^{19}$. Pese a lo anterior, los informes no son concluyentes entre la asociación de enemas de FS y NAP.

El diagnóstico se sospecha por la asociación temporal entre FSO e IRA, con un sedimento de orina anodino, algún grado de proteinuria o ambos.

No existe tratamiento específico para la NAP. La estrategia principal es la prevención. Esto incluye preferir aquellos laxantes osmóticos como PEG, ya que el riesgo de desarrollar falla renal es 12,6 veces menor versus el uso de $\mathrm{FSO}^{5}$.

En conclusión, la NAP impacta de forma 
aguda la función renal, y presenta un alto riesgo de desarrollo de enfermedad renal crónica. Para su prevención, es fundamental identificar a aquellos pacientes con mayor riesgo de desarrollar esta condición y evitar el consumo de laxantes fosfatados. El antecedente clínico de consumo de compuestos orales fosfatados y los hallazgos histológicos renales característicos, constituyen elementos fundamentales en la identificación y diagnóstico de esta patología.

\section{Referencias}

1. U.S. Food and Drug Administration. FDA Drug Safety Communication: FDA Warns Of Possible Harm From Exceeding Recommended Dose Of Over-The-Counter Sodium Phosphate Products To Treat Constipation. FDA 2014. Disponible en: www.fda.gov [Consultado el 7 de junio de 2020].

2. Loganathan A, Tan KS, Moore J, Oliver K. A case of acute phosphate nephropathy. Med J Aust. 2016; 204 (5): 183-183e1.

3. Schanz S, Kruis W, Mickisch O, Küppers B, Berg P, Frick B, et al. Bowel Preparation for Colonoscopy with Sodium Phosphate Solution versus Polyethylene Glycol-Based Lavage: A Multicenter Trial. Diagn Ther Endosc. 2008; 2008: 713521.

4. Lorenzo-Zúñiga V, Moreno-de-Vega V, Boix J. Preparation for colonoscopy: types of scales and cleaning products. Rev Esp Enferm Dig. 2012; 104 (8): 426-31.

5. Markowitz GS, Stokes MB, Radhakrishnan J, D’Agati VD. Acute phosphate nephropathy following oral sodium phosphate bowel purgative: an underrecognized cause of chronic renal failure. J Am Soc Nephrol. 2005; 16 (11): 3389-96.

6. Gonlusen G, Akgun H, Ertan A, Olivero J, Truong LD. Renal failure and nephrocalcinosis associated with oral sodium phosphate bowel cleansing: clinical patterns and renal biopsy findings. Arch Pathol Lab Med. 2006; 130 (1): 101-6.

7. Aasebø W, Scott H, Ganss R. Kidney biopsies taken before and after oral sodium phosphate bowel cleansing. Nephrol Dial Transplant. 2007; 22 (3): 920-2.

8. Beyea A, Block C, Schned A. Acute phosphate nephropathy following oral sodium phosphate solution to cleanse the bowel for colonoscopy. Am J Kidney Dis.
2007; 50 (1): 151-4.

9. Manterola P, Mur P, Vukusich A, Contreras L, Pezantes I, Boltansky A. Nefropatía asociada a fosfatos posterior a preparación para colonoscopia: dos casos Anátomo-Clínicos. Gastroenterol. latinoam. 2015; 26 (4): 209-12.

10. Khurana A, McLean L, Atkinson S, Foulks CJ. The effect of oral sodium phosphate drug products on renal function in adults undergoing bowel endoscopy. Arch Intern Med. 2008; 168 (6): 593-7.

11. Hurst FP, Bohen EM, Osgard EM, Oliver DK, Das NP, Gao SW, et al. Association of oral sodium phosphate purgative use with acute kidney injury. J Am Soc Nephrol. 2007; 18 (12): 3192-8.

12. Russmann S, Lamerato L, Marfatia A, Motsko SP, Pezzullo JC, Olds G, et al. Risk of impaired renal function after colonoscopy: a cohort study in patients receiving either oral sodium phosphate or polyethylene glycol. Am J Gastroenterol. 2007; 102 (12): 2655-63.

13. Brunelli SM, Lewis JD, Gupta M, Latif SM, Weiner MG, Feldman HI. Risk of kidney injury following oral phosphosoda bowel preparations. J Am Soc Nephrol. 2007; 18 (12): 3199-205.

14. Lieberman DA, Ghormley J, Flora K. Effect of oral sodium phosphate colon preparation on serum electrolytes in patients with normal serum creatinine. Gastrointest Endosc. 1996; 43 (5): 467-9.

15. Murer H, Hernando N, Forster L, Biber J. Molecular mechanisms in proximal tubular and small intestinal phosphate reabsorption (plenary lecture). Mol Membr Biol. 2001; 18 (1): 3-11.

16. Forster IC, Hernando N, Biber J, Murer H. Proximal tubular handling of phosphate: A molecular perspective. Kidney Int. 2006; 70 (9): 1548-59.

17. Markowitz GS, Nasr SH, Klein P, Anderson H, Stack JI, Alterman L, et al. Renal failure due to acute nephrocalcinosis following oral sodium phosphate bowel cleansing. Hum Pathol. 2004; 35 (6): 675-84.

18. Martin RR, Lisehora GR, Braxton M Jr, Barcia PJ. Fatal poisoning from sodium phosphate enema. Case report and experimental study. JAMA. 1987; 257 (16): 2190-2.

19. Schaefer M, Littrell E, Khan A, Patterson ME. Estimated GFR Decline Following Sodium Phosphate Enemas Versus Polyethylene Glycol for Screening Colonoscopy: A Retrospective Cohort Study. Am J Kidney Dis. 2016; 67 (4): 609-16.

20. Malberti F. Hyperphosphataemia: treatment options. Drugs. 2013; 73 (7): 673-88. 